\section{POSTER 018.} \section{Hazmat Preparedness: Sufficient or So-So?}

David C. Cone, MD, * Steven J. Davidson, $M D, M B A$

Department of Emergency Medicine, The Medical College of Pennsylvania, Philadelphia, Pennsylvania USA

Purpose: To examine hospital hazardous-materials preparedness. Methods: The safety officers of all acute-care hospitals in the five-county Philadelphia metropolitan region received surveys. Questions addressed the ability of EDs to safely decontaminate and treat chemically contaminated patients.

Results: Thirty-six of 58 hospitals $(62.1 \%)$ returned usable surveys. Of these, $25(69.4 \%)$ have a written ED hazmat plan, and $11(30.6 \%)$ conducted a drill of the plan in 1994. Nineteen $(52.8 \%)$ EDs have a specific treatment area for chemically contaminated patients. A stock of supplies for protecting the $\mathrm{ED}$ from secondary contamination is maintained by $16(44.4 \%)$. While $23(63.9 \%)$ EDs store personal protective equipment, most of these involve only gowns, gloves, and surgical masks; only 12 provide any type of respiratory protection. Seven respondents were certain that patients brought in by local EMS would have been decontaminated adequately in the field, eight stated that they believed or felt decontamination would be adequate, and 12 were concerned that field decontamination might not be adequate. Seventeen hospitals (47.2\%) reported treating one or more (mean $=2.4$ ) chemically contaminated patients in 1994. We believe the return rate reflects reluctance to commit hospital policies to paper. This was confirmed during telephone follow-up of nonrespondents; for example, one safety officer discussed hazmat principles for 40 minutes, but refused to complete the survey.

Conclusions: Hospital hazmat preparedness in this area varies tremendously. A significant proportion of EDs lack a written plan and equipment to allow the ED to safely and effectively handle the chemically contaminated patient. There is reluctance to discuss this topic.

\section{POSTER 019.}

\section{Work-Related Injuries Among Rural West} Virginia Emergency Medical Services Providers

Julia E. Martin, $M D$, Janet M. Williams, MD, Jennifer L. Bucklew, MS, John E. Prescott, MD

Department of Emergency Medicine/Center for Rural

Emergency Medicine, West Virginia University,

Morgantown, West Virginia USA

Purpose: To study mechanisms of occupational injury among EMS personnel in West Virginia(WV). To identify factors that influence the rate and types of injuries in order to predict and prevent future injuries.

Methods: A 16-item survey was distributed at the 1994 WV EMS Conference to 135 EMS providers representing the state's 13 regional EMS systems.

Results: Of the 135 surveys returned, two were incomplete and, therefore, omitted from the study ( $98 \%$ evaluated). Forty-six $(34.6 \%)$ reported occupational injuries during their career. Of those, $28(61 \%)$ stated that the injury occurred during direct patient care. Common injury mechanisms included: Overexertion, $24(52 \%)$; falls, five (11\%); and cut/puncture, three $(7 \%)$.

Back injury occurred most frequently, in 22 of the 46 (49\%). Lifting and carrying patients were the most common causes of injury. The second most common injury involved the head and face, accounting for five of $46(11 \%)$. The third most common type involved the hand in four of $46(9 \%)$.

Thirty-seven of the 46 (80\%) work-related injuries required medical attention or loss of work. Of these, $22(60 \%)$ were back injuries. Thirty-four of the $46(74 \%)$ injured workers had suggestions for how the injury could have been prevented, including use of protective equipment.

Conclusion: Work-related injuries, especially back injuries, are common among WV EMS providers. Back injuries tend to occur while lifting or carrying patients. This is where preventive efforts should be focused, emphasizing proper lifting techniques, personal physical fitness and use of personal protective gear. 